\title{
Erratum to: A new rhynchosaur from south Brazil (Santa Maria Formation) and rhynchosaur diversity patterns across the Middle-Late Triassic boundary
}

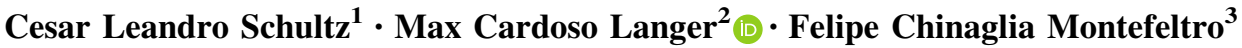

Published online: 12 September 2016

(C) Paläontologische Gesellschaft 2016

\section{Erratum to: Paläontol $\mathrm{Z}$ DOI 10.1007/s12542-016-0307-7}

In the original article the German abstract ("Kurzfassung") contained printing errors. The correct "Kurzfassung" is reproduced in full below. We apologize for this error.

Kurzfassung Der früher als „Mariante Rhynchosaur“ bezeichnete Rhynchosaurier wird hier anhand zweier Exemplare (ein unterkieferloser kompletter Schädel inklusive der ersten drei Halswirbel, sowie ein rechtes Maxillare und Dentale) formell als neue Gattung und Art beschrieben. Beide Exemplare stammen aus derselben Fundstelle im brasilianischen Bundesstaat Rio Grande do Sul, gefunden in Ablagerungen der mittel-triassischen
Santa Maria-Formation (Ladinium). Diagnostische Merkmale der neuen Gattung und Art umfassen den Kontakt zwischen Präfrontale und Postfrontale, ein Paar tiefer Frontalfurchen, sowie einen sehr tiefen Schädel. Eine neue phylogenetische Analyse offenbart das neue Taxon als Vertreter der Stenaulorhynchinae, einer relativ diversen Gruppe mittel-triassischer Rhynchosaurier, mit Nachweisen aus Indien, Ostafrika und Amerika. Es existieren Hinweise darauf, dass das Aussterben dieser monophyletischen Gruppe im Zusammenhang eines Faunenaustausches an der Ladinium/Karnium-Grenze stattfand, in dessen Verlauf diese durch die sehr viel häufigeren hyperodapedontinen Rhynchosaurier der späten Trias ersetzt wurden.
The online version of the original article can be found under doi:10.1007/s12542-016-0307-7.

\section{Max Cardoso Langer}

mclanger@ffclrp.usp.br

1 Departamento de Paleontologia e Estratigrafia, Instituto de Geociências, Universidade Federal do Rio Grande do Sul, Av. Bento Gonçalves 9500, Porto Alegre, RS 91540-000, Brazil

2 Departamento de Biologia, Faculdade de Filosofia Ciências e Letras de Ribeirão Preto, Universidade de São Paulo, Av. Bandeirantes 3900, Ribeirão Preto, SP 14040-901, Brazil

3 Departamento de Biologia e Zootecnia, Faculdade de Engenharia, Universidade Estadual Paulista, Passeio Monção 226, Ilha Solteira, SP 15385-000, Brazil 